\title{
Digitizing of UWB Signals Based on Frequency Channelization
}

\author{
Won Namgoong, Lei Feng \\ Department of Electrical Engineering \\ University of Southern California \\ namgoong@usc.edu, leifeng@usc.edu
}

\begin{abstract}
One of the primary challenges in the implementation of the UWB radio is the design of the analog-todigital converter (ADC). To relax the ADC requirements, the frequency channelized receiver is presented. Among the advantages of the frequency channelized receiver are the robustness to narrowband interference and the ease of designing the sample/hold circuitry. This paper summarizes some of our recent work on frequency channelized receiver for digitizing UWB signals.
\end{abstract}

\section{INTRODUCTION}

Although great headway has recently been made in efficient implementation of narrowband radios, the UWB signal has fundamentally different signal characteristics, making the use of existing receiver circuits and architectures ill suited. Compared to existing narrowband radios, the signal bandwidth of the UWB radio is at least an order of magnitude greater. Furthermore, the UWB radio must coexist with many other narrowband systems transmitting and receiving in the same bandwidth. Consequently, a UWB radio has intrinsically different sensitivity, selectivity, and bandwidth requirements, which imply radio circuit and architectural designs that are also substantially different.

There are numerous implementation challenges in the UWB radio. Chief among them is the extremely highsampling and large dynamic range requirements of the ADC. Other design challenges include the wideband amplification and the generation of narrow pulses at the transmitter. This paper focuses on relaxing the ADC requirements in the UWB radio.

The bandwidth and dynamic range requirements of the ADC appear to have led to two alternative development paths. In the first, the UWB system is scaled down to operate at a greatly reduced bandwidth, compromising the benefits of the UWB radio. An example of such a system is the time-frequency interleaved (TFI) OFDM radio pro-

This work was supported in part by the Army Research Office under contract number DAAD19-01-1-0477 and National Science Foundation under contract number ECS0134629 . posed as a possible candidate for the 802.15 WPAN standard [1]. In the other development path, receiver functions such as correlation are performed in the analog domain before digitizing at a much reduced sampling frequency [2]. Such analog receivers are less flexible and suffer from circuit mismatches and other non-idealities. These circuit non-idealities limit the number of analog correlators that can be practically realized on an integrated circuit (IC). Since many correlators are required to exploit the diversity available in an UWB system, analog receivers do not perform well. These circuit non-idealities also preclude the use of sophisticated narrowband interference suppression techniques, which can greatly improve the receiver performance in environments with large narrowband interferers such as in UWB systems.

To achieve high reception performance, therefore, the UWB signal needs to be digitized at the signal Nyquist rate of several gigahertz, so that all of the receiver functions are performed digitally. In addition, as digital circuits become faster and denser with constant scaling of CMOS technology, simplifying the analog circuits as much as possible and distributing as much of the analog operations to the digital domain should prove more beneficial.

\section{DIGITAL RECEIVER ARCHITECTURES}

Since designing a single ADC to operate at the signal Nyquist rate is not practical, parallel ADC architectures with each ADC operating at a fraction of the effective sampling frequency need to be employed. To sample at a fraction of the effective sampling frequency, the received UWB signal can be channelized either in the time or frequency domain. An approach that has been used in high-speed digital sampling oscilloscopes is to employ an array of $M$ ADCs each triggered successively at $1 / M$ the effective sample rate of the parallel ADC. A fundamental problem with an actual implementation of such time-interleaved architecture is that each ADC sees the full bandwidth of the input signal. This causes great difficulty in the design of the sample/hold circuitry. Furthermore, in the presence of strong narrowband interferers, each ADC requires an impractically large dynamic range to resolve the signal from the narrowband interferers. 
Instead of channelizing by time-interleaving, the received signal can be channelized into multiple frequency subbands with an ADC in each subband channel operating at a fraction of the effective sampling frequency [3]. As designing bandpass filters with high center frequency is difficult, channelization can be achieved using a bank of $M_{\mathrm{s}}$ mixers operating at equally spaced frequencies and $M_{\mathrm{s}}$ lowpass filters to decompose the analog input signal into $M_{\mathrm{S}}$ subbands. A block diagram of such a receiver is shown in Fig. 1. A total of $M=2 M_{\mathrm{s}}-1$ ADCs each operating at $f_{\mathrm{s}}$ is employed to achieve an effective sampling frequency of $M f_{\mathrm{s}}$. In addition to obviating the need to design high frequency bandpass filters, channelizing the received signal using this approach greatly relaxes the design requirements of the sample/hold circuitry. The sample/hold circuitry in this lowpass channelized architecture sees only the bandwidth of the subband signal; whereas in the bandpass channelization approach, the sample/hold circuitry sees the uppermost frequency in the high-frequency subbands.

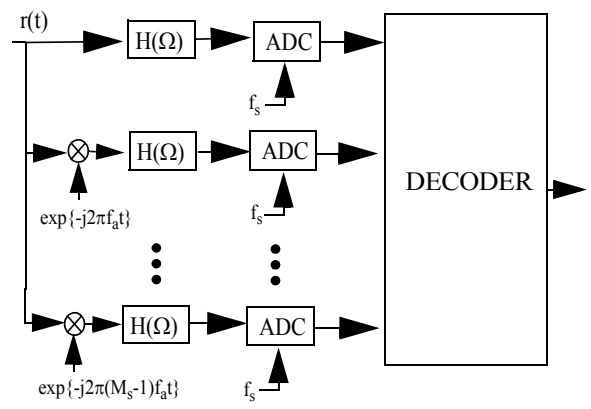

Fig. 1 Frequency channelized receiver

When no narrowband interferers (NBI) are present, the performance of the time-interleaved and frequency channelized receivers are nearly identical. However, when finite resolution ADC's are employed in the presence of large narrowband interferers, the frequency channelized receiver significantly outperforms the time-interleaved receiver. As shown in [3], for example, an SNR difference of approximately $20 \mathrm{~dB}$ is observed when 4-bit ADCs are used compared to the time-interleaved receiver assuming MMSE detectors are employed.

This large performance difference arises because the frequency channelization process better isolates the effects of the narrowband interferer by raising the quantization noise floor mostly in the subband channels containing the interferers. Since significant interference noise is already present in these subband channels, the additional quantization noise does not greatly increase the total noise power relative to the signal power. By contrast, a narrowband interferer in the time-interleaved receiver increases the quantization noise floor across the entire signal spectrum. Thus, even in frequencies with no interference, the quantization noise floor is significantly raised relative to the sig- nal power, resulting in large overall performance degradation. The ability to isolate the narrowband interferer significantly improves the performance of the frequency channelized receiver compared to the timeinterleaved receiver, especially when low resolution ADCs are employed for complexity reasons.

The frequency channelized receiver requires accurate knowledge of the transfer functions of the analog filters, which may be unavailable in practice because of the variations resulting from temperature and fabrication process uncertainties. Although adaptive digital filters can be employed, the convergence speed may be slow because of the increased number of parameters to estimate and the difficulty in estimating the overlapping frequency components between subband channels. Despite the numerous implementation advantages, the slow convergence speed in the channelized receiver is problematic as the UWB radio must operate in the rapidly time varying wireless environment.

In several UWB communication systems, however, the channelized receiver can be made to achieve fast convergence speed. Two such examples are cosidered. The first is the generalized transmitted reference (TR) system using an oversampled channelized receiver for low data rate systems. The other is a maximally decimated channelized receiver for high data rate communications based on cyclic prefix single-carrier (CP-SC) systems [4].

\section{GENERALIZED TR SYSTEMS}

The UWB signal pulses are transmitted in fixed time intervals where the interval is sufficiently large to prevent interference between consecutive pulses. Assuming a block of $N_{b}$ pulses are transmitted, the received UWB signal $r(t)$, which is real, is

$$
r(t)=\sum_{k=0}^{N_{b}-1} a_{k} s(t-k T)+v(t)
$$

where $a_{k}$ is the $k$ th transmitted antipodal symbol, $T$ is the time interval between consecutive pulses, $s(t)$ is the received signal pulse response, and $v(t)$ is the additive white Gaussian noise (AWGN). In each transmission block, we assume that the first $N_{r}$ pulses are reference pulses and are not data modulated, i.e., $a_{k}=1$ for $0 \leq k<N_{r}$. The remaining pulses are data pulses. In the generalized TR system, the reference pulses are averaged to approximate the matched filter response, which is then used to decode the remaining data pulses.

\subsection{Channelized TR Receivers}

Conceptually, the simplest method for detecting the received signal is to first perfectly reconstruct the received signal then process it as in a full-band receiver. If the equivalent analysis filters, which are implemented as a 
combination of mixer $\left\{e^{-j 2 \pi m f_{a} t}\right\}$ and lowpass filter $H(\Omega)$ in Fig. 1, are designed to satisfy the power complementary condition (as described shortly), the perfect reconstruction (PR) synthesis filters are the time-reversed complex conjugates of the analysis filters [5]. Since the matched filter is also the time-reversed complex conjugate of the propagation channel, the matched filter and the synthesis filter can be combined and the estimate of the optimal pulse response for detection can be obtained by simply averaging the combined responses of the propagation channel and the analysis filters. The detection process then simply consists of averaging the reference pulse responses in each of the subband channels indepedently and using them to correlate the data pulses [6].

A $M_{s}$ subband frequency channelized receiver for TR systems is shown in Fig. 2. The received signal $r(t)$ is passed through a set of equally spaced mixers $e^{-j 2 \pi m f_{a} t}$, $m \in\left\{0,1, \ldots, M_{s}-1\right\}$. In each subband, the signal is filtered by a continuous-time analysis lowpass filter $H(\Omega)$, sampled and digitized by the ADC at a rate of $1 / T_{S}$, filtered by the discrete-time subband filter $W(\omega)$, and correlated with the conjugate of $\hat{s}_{m}[l]$, which is the estimate of the $m$ th subband pulse response $s_{m}[l]$. The scaling factor $A_{m}$ in each subband channel models the effect of the automatic gain controller (AGC). The power complementary condition of the analysis filters can be satisfied by designing the digital filter $W(\omega)$ to satisfy the power complementary condition and ensuring (by sufficiently oversampling) that the overall amplitude response of $H(\Omega)$ and $W(\omega)$ is approximately $|W(\omega)|$ despite uncertainties in the analog circuitries from process and temperature variations.

The slicer input $y_{k}$ is a linear combination of subband correlations $y_{k, m}$

$$
y_{k}=\sum_{m=0}^{M-1} \alpha_{m} y_{k, m}
$$

As shown in [7], the weighting coefficients $\alpha_{m}$ should be the inverse of the subband noise power, which can be readily estimated by computing the variance at the output of $W(\omega)$ based on the first $N_{r}$ training symbols. This weighting scheme yields near optimal detection in the generalized TR system regardless of the input noise statistics and the AGC scaling factors. For example, when the input noise is colored or NBI is present, this weighting scheme can be shown to perform joint noise whitening and matched filtering operations [7].

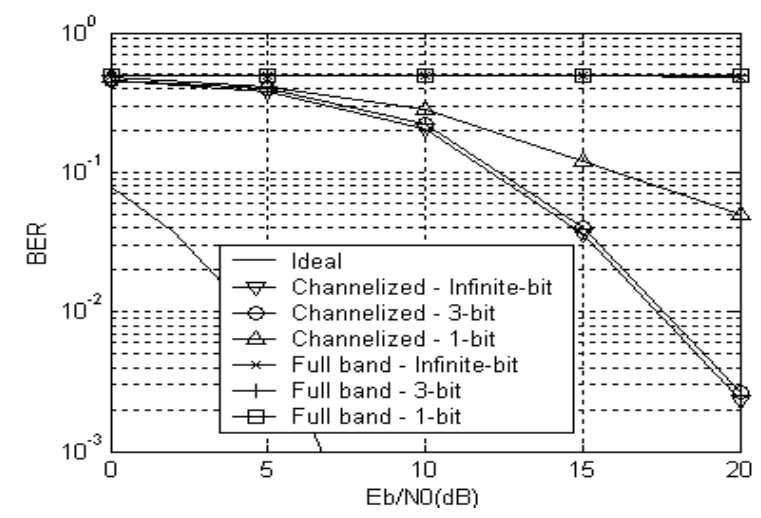

Fig. 3 NBI with $S I R=-5 d B$

\subsection{Performance Results}

Assuming the second derivative of a gaussian pulse and the CM1 channel model recommended by the IEEE 802.15.3a committee, the performance of a channelized receiver is compared to an ideal full-band receiver in a TR system. The effective sampling frequency for both receiver is $1 / T_{e}=3.5 \mathrm{GHz}$. In the channelized receiver, seven ADCs are employed with each operating at $700 \mathrm{MS} / \mathrm{s}$, and $H(\Omega)$ is a fourth order Butterworth filter.

In Fig. 3, the effect of the narrowband interference (NBI) on BER is shown assuming signal-to-interference ratio (SIR) of $-5 \mathrm{~dB}$. The first four reference pulses in a block of 100 symbols is used to estimate the time varying $s_{m} *[l]$. The performance of an ideal matched filter receiver with perfect knowledge of the received signal pulse is shown as the lower bound. Regardless of $E_{b} / N_{0}$ values, the full band receiver ceases to function, but the channelized receiver performs well even when low resolution ADCs are employed.

\section{CYCLIC PREFIX SINGLE-CARRIER SYSTEM}

In a CP-SC system, the UWB transmitter sends blocks of consecutive data pulses each with cyclic prefix (CP). The propagation channel $\mathbf{P}$ can then be modeled as a circulant

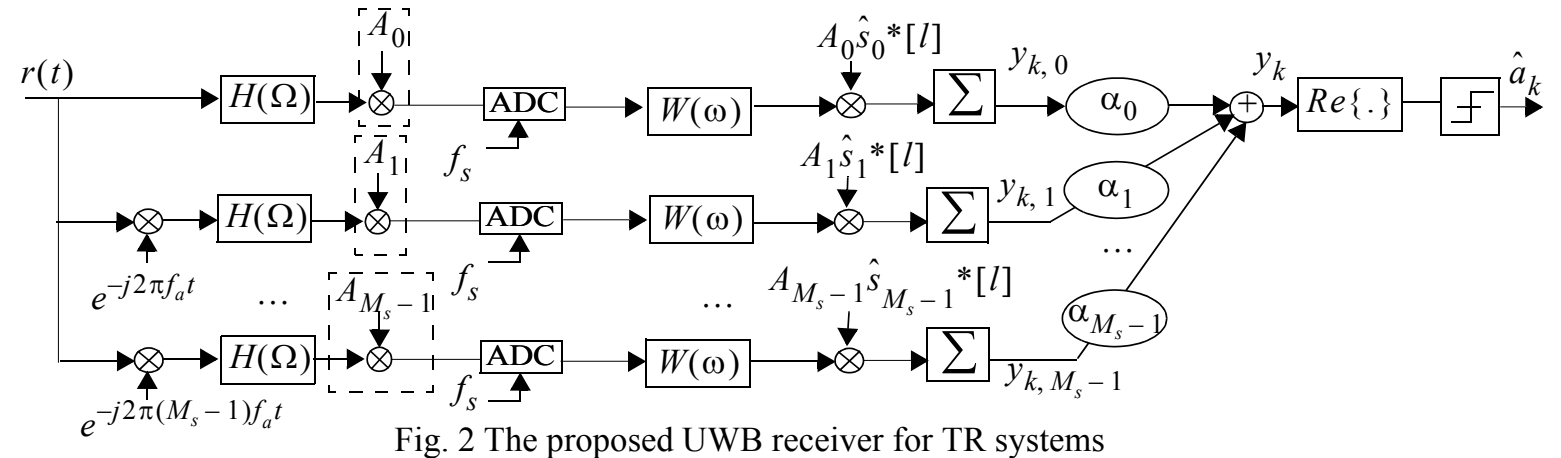

Fig. 2 The proposed UWB receiver for TR systems 
matrix (CM), which can be decomposed as a diagonal matrix $\Lambda$ and FFT/IFFT matrices $\left(\mathbf{F}^{-1}\right.$ and $\left.\mathbf{F}\right)$ [4].

$$
\mathbf{P}=\mathbf{F}^{-1} \Lambda \mathbf{F}
$$

When a frequency channelized receiver is used in CP$\mathrm{SC}$ system, the frequency channelizer can be modeled as a block circulant matrix (BCM) H. All BCM matrices can be decomposed into two fixed DFT related matrices $\left(\mathbf{F}_{B}^{-1}\right.$ and $\mathbf{F}_{B}$ ) and a block diagonal matrix $\mathbf{D}$ [8].

$$
\mathbf{H}=\mathbf{F}_{B}^{-1} \mathbf{D} \mathbf{F}_{B}
$$

Because the impulse response of the subband filters are modulated versions of a prototype filter, $\mathbf{D}$ can be further decomposed into a fixed DFT related matrix $\mathbf{F}_{D}$ and a diagonal matrix $\mathbf{C}$. The combined response of the propagation channel and the channelier is described as

$$
\mathbf{H}=\mathbf{F}_{B}^{-1} \mathbf{F}_{D} \mathbf{C} \mathbf{F}_{B} \mathbf{F}^{-1} \Lambda \mathbf{F}
$$

In receiver equalization, the two diagonal matrices could be implemented as one-tap equalizers [9]. Detection can then be achieved by adapting the cascaded one-tap equalizers and performing several DFT/IDFT related matrices.

A block diagram of the detection process in the frequency channelized receiver is illustrated in Fig. 4. After forming a vector $\mathbf{x}$ with the samples from all the subbands, $\mathbf{x}$ is multiplied by a set of DFT/IDFT related matrices and diagonal matrices, $\mathbf{C}^{-\mathbf{1}}$ and $\Lambda^{-1}$.

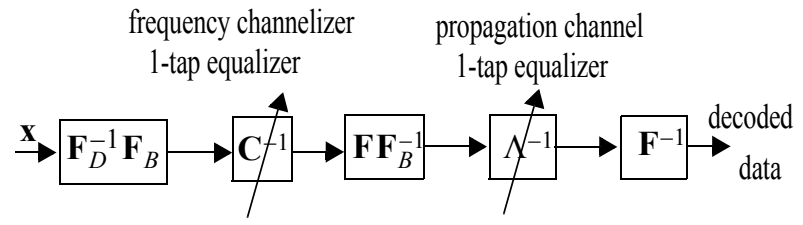

Fig. 4 Detection block diagram in CP system.

The adaptive algorithm based on the mean squared error (MSE) criterion is derived in [9]. Since the receiver can adaptively compensate the propagation channel and the channelizer separately, each of which vary at vastly different rates, the one-tap equalizers for the frequency channelizer can be fixed or updated very slowly to track variations in the analog analysis filters after initial convergence. The adaptive receiver then operates as if its input is from an ideal single $\mathrm{ADC}$, resulting in convergence performance close to an ideal CP-SC system. The performance is illustrated in Fig. 5, which shows that the channelized receiver after initial convergence performs comparably to an ideal full band receiver.

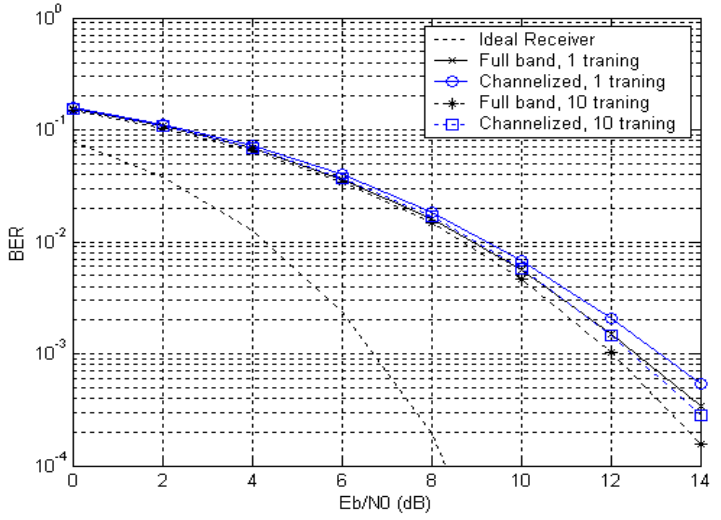

Fig. 5 BER Performance

\section{REFERENCES}

[1] A. Batra, etc. "Design of a multiband OFDM system for realistic UWB channel environments," Microwave Theory and Tech., IEEE Trans. on, vol. 52, pp. 2123 - 2138, Sept. 2004

[2] R. D. Wilson and R. A. Scholtz, "Comparison of CDMA and modulation schemes for UWB radio in a multipath environment," GLOBECOM 2003, vol. 2, pp. 754 - 758

[3] W. Namgoong, "A Channelized Digital Ultra-Wideband Receiver," IEEE Trans. Wireless Comm., vol. 2, pp. 502510, May 2003

[4] J. Louveaux, L. Vandendorpe and T. Sartenaer, "Cyclic Prefixed Single Carrier and Multicarrier Transmisson: Bit Rate Comparison," IEEE Comm. Letters, vol. 7, NO. 4, pp.180-182, Apr. 2003

[5] R. E. Crochire and L. R. Rabiner, "Multirate Digital Signal Processing," Prentice Hall, 1983

[6] L. Feng and W. Namgoong, "An oversampled channelized UWB receiver," Joint UWBST \& IWUWBS. 2004 International Workshop on, May 2004, pp. 410 - 414

[7] L. Feng and W. Namgoong, "Oversampled Channelized Receiver for Transmitted Reference UWB System in the Presence of Narrowband Interference," IEEE Signal Processing Systems, 2004, pp. 48 - 52

[8] R. Vescovo, "Inversion of Block-Circulant Matrices and Circular Array Approach," IEEE Trans. on Antennas and Propagation, vol. 45, NO. 10, pp.1565-1567, Oct. 1997

[9] L. Feng and W. Namgoong, "An Adaptive Maximally Decimated Channelized UWB Receiver with Cyclic Prefix", ICC 2005 